\title{
A Differentiated Service Model for Enhanced Distributed Channel Access (EDCA) of IEEE 802.11e WLANs
}

\author{
Jeng Farn Lee • Wanjiun Liao • Meng Chang Chen
}

Published online: 15 December 2006

(C) Springer Science + Business Media, LLC 2006

\begin{abstract}
In this paper, we propose a new differentiated service model, referred to as Differentiated Service-EDCA (DS-EDCA), for the Enhanced Distributed Channel Access (EDCA) of IEEE 802.11e wireless local area networks (WLANs). With DS-EDCA, both strict priority and weighted fair service can be provided. The strict priority service is provided for high priority traffic through carefully setting the EDCA parameter sets of lower priority traffic; the proportional fairness service is enabled by determining the backoff intervals according to the distributed scheduling discipline (DFS). We also propose a hierarchical link sharing model for IEEE 802.11e WLANs, in which AP and mobile stations are allocated different amounts of link resource. The performance of DS-EDCA and EDCA is compared via ns-2 simulations. The results show that DS-
\end{abstract}

A preliminary version of this paper has been published in Proc. IEEE Globecom 2005.

W. Liao $(\bowtie)$

BL-422, Department of Electrical Engineering, National Taiwan University, Taipei 106, Taiwan e-mail:wjliao@cc.ee.ntu.edu.tw

\section{J. F. Lee}

BL-608, Department of Electrical Engineering,

National Taiwan University, Taipei 106, Taiwan

e-mail: kunimi@iis.sinica.edu.tw

\section{J. F. Lee}

R614, Institute of Information Science,

Academia Sinica, Taipei 11529, Taiwan

W. Liao

Graduate Institute of Communication Engineering,

National Taiwan University, Taipei, Taiwan

M. C. Chen

R802, Department of Electrical Engineering,

National Taiwan University, Taipei 106, Taiwan

e-mail: mcc@iis.sinica.edu.tw
EDCA outperforms the original EDCA in terms of its support for both strict priority and weighted fair service. More importantly, DS-EDCA can be easily implemented, and is compatible to the IEEE 802.11 Standard.

Keywords EDCA $\cdot 802.11 \mathrm{e} \cdot$ strict priority $\cdot$ weighted fair service $\cdot$ hierarchical link sharing

\section{Introduction}

In this paper, we propose a differentiated service model for the Enhanced Distributed Channel Access (EDCA) of IEEE 802.11e WLANs. The EDCA of 802.11e, an enhanced version of $802.11 \mathrm{DCF}$, provides priority-based service for different kinds of applications [1]. With EDCA, each station can implement up to four access categories (ACs), which are $\mathrm{AC}_{-} \mathrm{VO}, \mathrm{AC}_{-} \mathrm{VI}, \mathrm{AC}_{-} \mathrm{BE}$, and $\mathrm{AC}_{-} \mathrm{BK}$, corresponding to voice, video, best effort, and background traffic, respectively. Each $\mathrm{AC}$ is associated with one backoff entity, which operates in a way similar to that implemented in legacy DCF. Each backoff entity is associated with a set of AC-specific parameters called the EDCA parameter set consisting of Arbitrary Inter-Frame Space Number (AIFSN[AC]), minimum contention window (CWmin[AC]), and maximum contention window (CWmax[AC]). The AIFSN[AC] is used to determine the duration of Arbitrary IFS (AIFS[AC]) according to the following equation:

$$
\begin{aligned}
\operatorname{AIFS}[\mathrm{AC}]= & \text { SIFS }+\operatorname{AIFSN}[\mathrm{AC}] \\
& \times \text { aslotTime, } \operatorname{AIFSN}[\mathrm{AC}] \geq 2,
\end{aligned}
$$

where aslotTime is the duration of a slot. Different backoff entities are then prioritized according to the values of their AC parameter sets. 
Wireless Multimedia (WMM) [2] is a subset of IEEE $802.11 \mathrm{e}$ Standard, and is motivated by the need to provide QoS implementation in the industry. In WMM, only EDCA is defined. To support real-time applications via EDCA, other advanced QoS features, such as ensuring the minimum guaranteed or expected throughputs, and allowing service amounts in proportion to the demands, should be provided. Specifically, it is desirable to implement both strict priority and weighted fair service in EDCA. Implementing the former is relatively straightforward, as EDCA by itself supports priority-based service. To provide the latter, we require fair queuing in which different flows contending for a shared link are allocated bandwidth in proportion to their "weights." The typical approach to implement fair queuing in existing work is to emulate the Generalized Processor Sharing (GPS) service discipline [3]. GPS is a fluid system in which traffic is infinitely divisible and all traffic streams can receive service simultaneously. In GPS, each flow $i$ is assigned a positive real number $\phi_{i}$, which indicates the weight of flow $i$ for sharing the channel capacity. Let $W_{i, \mathrm{GPS}}\left(t_{1}, t_{2}\right)$ denote the amount of workload received by flow $i$ over time interval $\left[t_{1}, t_{2}\right]$. A GPS server guarantees the following equation for each flow which is continuously backlogged over the interval $\left[t_{1}, t_{2}\right]$,

$\frac{W_{i, \mathrm{GPS}}\left(t_{1}, t_{2}\right)}{W_{j, \mathrm{GPS}}\left(t_{1}, t_{2}\right)} \geq \frac{\phi_{i}}{\phi_{j}} i, j=1,2, \ldots n$.

A flow is backlogged if it has packets waiting for transmission in the queue on the host or the access point (AP) or its packet is being transmitted. Let $B(t)$ denote the set of the backlogged flows at time $t$, and $C$ be the link capacity. The service rate $r_{-} \operatorname{GPS}_{i}(t)$ of each backlogged flow $i$ at time $t$ can be expressed by

$r_{-} \operatorname{GPS}_{i}(t)=\frac{\phi_{i}}{\sum_{j \in B(t)} \phi_{j}} C$.

The major challenge of providing fair queuing in WLANs is in that service provisioning must be fully distributed. This renders existing centralized mechanisms (e.g., [4] for wired networks and [5] for wireless networks in which base stations are involved) inappropriate. Some scheduling disciplines have been proposed for weighted fairness at the link or MAC layer of legacy 802.11 DCF, including Distributed Fair Queuing (DFS) [6], Prioritybased fair Medium Access Control (P-MAC) [7], and Distributed Deficit Round Robin (DDRR) [8]. The typical approach of these efforts is to tune one of the three 802.11 MAC parameters, i.e., Backoff Interval (BI), Contention Window (CW), and Inter-Frame Space (IFS), based on certain fair queuing mechanisms. These efforts, however, all aim to provide station-based service differentiation, rather than AC-based QoS as provided in EDCA. In [9], Adaptive Fair Enhanced DCF (AFEDCF) is proposed to implement fairness among flows of the same priority for 802.11e EDCA. However, AFEDCF cannot provide weighted fair service among different ACs and thus is not a proportional fairness mechanism. In [10], an IFS-based service differentiation mechanism is presented to provide AC-based proportional fairness service for 802.11e EDCA. However, that mechanism has some limitations, such as the weights of different ACs cannot be assigned arbitrarily and the total number of stations is limited. This calls for a more flexible differentiated service model for EDCA and motivates our work.

In this paper, we propose a differentiated service model, called Differentiated Service-EDCA (DS-EDCA), to support both strict priority and weighted fair service for IEEE 802.11e EDCA WLANs. In DS-EDCA, the resource is first allocated to flows of higher priority. The remaining bandwidth is then shared proportionally among the other service priorities according to their assigned weights. A hierarchical link sharing model for DS-EDCA is also proposed, which allows efficient sharing of downlink and uplink traffic among AP and stations. Based on the hierarchical link sharing model, network managers can allocate different amounts of link resource to downlink and uplink traffic based on some performance metrics. The performance of DS-EDCA is evaluated via ns-2 simulations. The results show that DS-EDCA outperforms EDCA in terms of the support of both strict priority and weighted fair services for IEEE 802.11e WLANs.

The rest of the paper is organized as follows. We review the related work for weighted fair scheduling in 802.11 WLANs in Section 2. In Section 3, the operations of DSEDCA are described and a hierarchical link sharing model in IEEE 802.11e WLANs is proposed. In Section 4, the performance of the proposed mechanism is evaluated via ns2 simulations. Finally, the paper is concluded in Section 5.

\section{Related work on weighted fair scheduling in 802.11}

\section{(1) $D F S$}

DFS is designed to emulate Self-Clocked Fair Queuing (SCFQ) [11] in a distributed manner. In SCFQ, a virtual clock is maintained by the central coordinator in the system. Let $v(t)$ denote the virtual clock at real time $t, P_{i}^{k}$ be the $k$ th packet of flow $i$, and $A_{i}^{k}$ be the real arrival time of $P_{i}^{k}$. Let $L_{i}^{k}$ denote the packet size of $P_{i}^{k}$ and $\phi_{i}$ the assigned weight of flow $i$. A start tag $S_{i}^{k}$ and a finish tag $F_{i}^{k}$ are associated with each packet $P_{i}^{k}$, given by

$$
\begin{aligned}
S_{i}^{k} & =\max \left\{v\left(A_{i}^{k}\right), F_{i}^{k-1}\right\} \\
F_{i}^{k} & =S_{i}^{k}+\frac{L_{i}^{k}}{\phi_{i}}
\end{aligned}
$$

The system virtual clock is initialized to 0 , and is updated to be the finish tag of the packet being transmitted. In 
SCFQ, packets are transmitted in increasing order of finish tags. Ties are broken arbitrarily.

To emulate SCFQ in which the frame with the smallest finish tag is transmitted first, each transmitted frame in DFS is stamped with a finish tag, based on which the BI of each competing mobile station is chosen. The backoff interval $\mathrm{BI}_{i}$ for each wireless station $i$ is expressed by

$\mathrm{BI}_{i}=\left\lfloor\left\lfloor\right.\right.$ Scaling_Factor $\left.\left.\times \frac{L_{i}}{\phi_{i}}\right\rfloor \times \rho\right\rfloor$,

where $L_{i}$ is the size of the head of line frame, $\phi_{i}$ is the assigned weight of station $i$, and $\rho$ is a random variable uniformly distributed in $[0.9,1.1]$ for preventing collisions. The ratio between $L_{i}$ and $\phi_{i}$ in Eq. 5 is based on the finish tag in Eq. 4, and the Scaling_Factor allows the choice of an appropriate scale for the backoff interval in DFS. DFS requires a complicated backoff interval mapping scheme to improve its throughput performance as the duration of the backoff interval is inversely proportional to the weight of a station.

\section{(2) $P-M A C$}

In P-MAC, the weighted fairness is achieved by adjusting the contention window $(\mathrm{CW})$ of each station as follows:

$\mathrm{CW}_{j}=\frac{\mathrm{CW}_{1}-1}{\phi_{j}}+1$,

where $\mathrm{CW}_{j}$ and $\phi_{j}$ are the $\mathrm{CW}$ and the weight of stations of class $j$, respectively, assuming the weights of all stations of class 1 are $1 . \mathrm{CW}_{1}$ is an optimal value properly selected to reflect the number of stations contending for the wireless medium such that P-MAC can maximize the aggregate throughput. However, P-MAC requires that each wireless station keeps sensing the channel and monitoring the activities on the wireless medium such that each station can learn (1) the traffic class to which a successfullytransmitted frame belongs and (2) the number of wireless stations in each traffic class. Moreover, the proportional fairness service achieved by P-MAC is through controlling the medium access probability of each station but without considering the frame size. This may lead to unfairness when the frame size is not fixed.

\section{(3) $D D R R$}

DDRR is based on Deficit Round Robin (DRR) [12] to translate user requirements into the IFS parameter of 802.11 MAC. Each station $i$ is allocated a service quantum $Q$ bits every $T_{i}$ seconds such that $Q / T_{i}$ is the desired throughput. The Deficit Counter (DC) of each station $i$ (denoted by $\mathrm{DC}_{i}$ ) is increased at a rate of $Q$ bits every $T_{i}$ seconds, and is decreased by the size of the frame when a frame is transmitted. The deficit counter value is then mapped to an appropriate IFS value at each station. At time $t$, the IFS for wireless station $i$ can be expressed by:
$\mathrm{IFS}_{i}=\mathrm{DIFS}-\alpha \frac{\mathrm{DC}_{i}(t)}{Q} \times \operatorname{random}(1.0, \beta)$,

where $\alpha$ is a scaling factor to ensure $\operatorname{IFS}_{i}$ falling between PIFS and DIFS, and $\beta$ is a value larger than 1. In DDRR, unlike DFS and P-MAC, no backoff algorithm is employed. Collisions are avoided by randomizing the IFS values. This is accomplished by multiplying $\alpha$ by a random number between 1 and $\beta$ as in Eq. 7 .

DDRR, however, has a potential fairness problem. This problem happens when a station which has accumulated a high DC value (due to transmitting at a rate lower than the desired throughput) starts transmission at a high rate. This may cause starvation to other stations because, in DDRR, the deficit counters of stations continue to accumulate when stations are inactive, and at the time they start transmissions, their IFS values are determined according to the accumulated deficit counters, without reference to the normalized (global) service amount in the system. Moreover, when the deficit counters of some stations become zero due to their sending rates exceeding their assigned target rates, these stations will share the link capacity equally. This further renders the system unable to provide proportional fairness. Improper settings of parameters may also degrade the performance of DDRR. Most of all, DDRR has an implementation problem due to its lack of consideration of the physical layer limitations. With DDRR, each node contends for the channel with a different IFS value bounded by PIFS and DIFS. Since there is only one slot time difference between a PIFS and a DIFS, and the slot time duration in IEEE 802.11 is determined by the physical layer, which corresponds to the minimum carrier sensing time period plus the transmission/receiving turnaround time period, stations transmitting at the same time slot will collide no matter which station starts transmission earlier at that slot.

\section{Differentiated service-enhanced distributed channel access (DS-EDCA)}

In this section, we describe the proposed mechanism called DS-EDCA for IEEE 802.11 WLANs. With DS-EDCA, 802.11e EDCA is enhanced by using Distributed Fair Scheduling (DFS) in each backoff entity to provide weighted fair service for flows of different ACs. The strict priority is ensured by carefully assigning the EDCA parameter sets of lower priority traffic.

\subsection{Proportional fairness service}

To provide proportional fairness service, each $\mathrm{AC}$ is assigned a positive real number $\phi[\mathrm{AC}]$ indicating the 
weight of sharing the channel capacity. The calculation of BI (Backoff-Interval) of the head-of-line packet is based on Eq. 5 in DFS. Note that DFS may need a backoff interval mapping scheme to improve the throughout performance as the duration of the backoff interval is inversely proportional to the weight of a station. Any increasing function can be used as the mapping scheme of DFS, and the following square-root mapping scheme proposed in [6] is used in DS-EDCA.

$$
\begin{aligned}
\mathrm{BI}_{i} & =\psi(\Delta) \\
& =\left\{\begin{array}{cc}
\Delta & \text { if } \Delta<\text { Threshold } \\
\lfloor\sqrt{\text { Threshold } \times \Delta}\rfloor, & \text { otherwise }
\end{array}\right.
\end{aligned}
$$

where $\Delta$ denotes the backoff interval obtained in Eq. 5 and Threshold is a constant parameter.

\subsection{Strict priority service}

To provide strict priority, higher priority frames must be transmitted before the lower-priority ones. In other words, the AIFS values of the lower priority must be larger than that of the higher priority plus its maximum contention window, i.e.,

$$
\operatorname{AIFSN}[j] \geq \operatorname{AIFSN}[i]+\mathrm{CW}_{\max [i]} \text { if } j<i
$$

To sum up, with DS-EDCA, each AC is associated with a backoff entity and an EDCA parameter set, i.e., AIFSN [AC], CWmin[AC], and CWmax[AC], but the value of the AIFSN[AC] is now determined by Eq. 9. The backoff intervals of strict priority classes are the same as the value defined in EDCA and the backoff intervals of proportional classes are calculated as in Eq. 5.

\subsection{Hierarchical link sharing architecture}

In 802.11e WLANs, the backoff entities of all mobile stations and the AP contend for the wireless bandwidth in the same way. Since the downlink traffic to all mobile stations must pass through the AP, the amount of downlink traffic may be several times the amount of uplink traffic (from mobiles to AP) sent by each mobile station. As a result, the downlink traffic will be dropped at the AP when the uplink traffic is heavy due to the input rate being larger than the service rate. For example, if there are four pairs of VoIP sessions between mobile stations, the aggregated downlink VoIP traffic is eight times of the traffic in the uplink direction for each mobile station. This implies that the throughput of the downlink VoIP traffic must be eight times of the throughput for each mobile station in the uplink direction in order to ensure the demanded QoS of VoIP. This problem has been ignored in all existing work. In this subsection, we will propose a hierarchical link sharing architecture for 802.11e WLANs, based on which the proposed DS-EDCA mechanism can provide the desired service for different classes of traffic.

Figure 1 shows the link sharing hierarchy of WLANs with respect to the uplink and downlink traffic. The AP first contends for the downlink bandwidth with all mobile stations contending for the uplink traffic, and then distributes the downlink resource to all mobile stations waiting for downlink traffic. The resource may be distributed to each station equally, or according to their pre-assigned weights if a weighted-fair discipline is adopted for the downlink traffic. Figure 2 shows the system architecture of the 802.11e AP with a centralized queuing discipline adopted by each AC. Before entering the backoff entity of each AC in the AP, the frames to each mobile station are scheduled based on the respective queuing discipline. Then, the backoff entities of AP contend for the downlink bandwidth with the backoff entities of all mobile stations contending for the uplink bandwidth. Therefore, the values assigned to the EDCA parameter sets of AP should be different from those of the mobile stations if we want to provide differentiated service among downlink and uplink traffic. Moreover, if there is some data traffic such as FTP or Web browsing flows in the system, the link bandwidth in both directions should be assigned to cater to real-time traffic (say, VoIP) first so as to meet the more stringent delay and delay jitter requirements of such this real-time traffic. Suppose there are four pairs of VoIP flows in the system and each VoIP session needs a rate of $\mathrm{V}$ bps to meet the desired QoS. Let AC_VO denote the VoIP traffic, and AC_BE, the data traffic. For AC_VO (i.e., high priority traffic), the backoff entity of AP should share $8 \mathrm{~V}$ bps and that of each mobile station, $\mathrm{V}$ bps. The remaining bandwidth is then shared by the uplink and downlink data traffic (low priority traffic). In general, if there are $n$ uplink data flows, and the weights of uplink and downlink data traffic are maintained in the ratio of $j: k$. The weights of the backoff entities of low priority ACs (AC_BE) for AP and each mobile station should be set to $h: i$, where $h: n^{*} i=k: j$, i.e.,

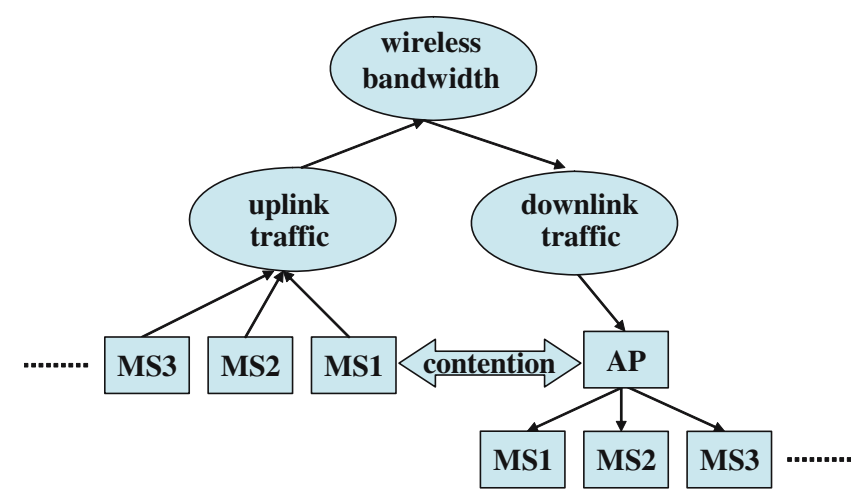

Figure 1 The hierarchical link sharing architecture in WLANs 
Figure 2 System design for the DS-EDCA enabled Access Point

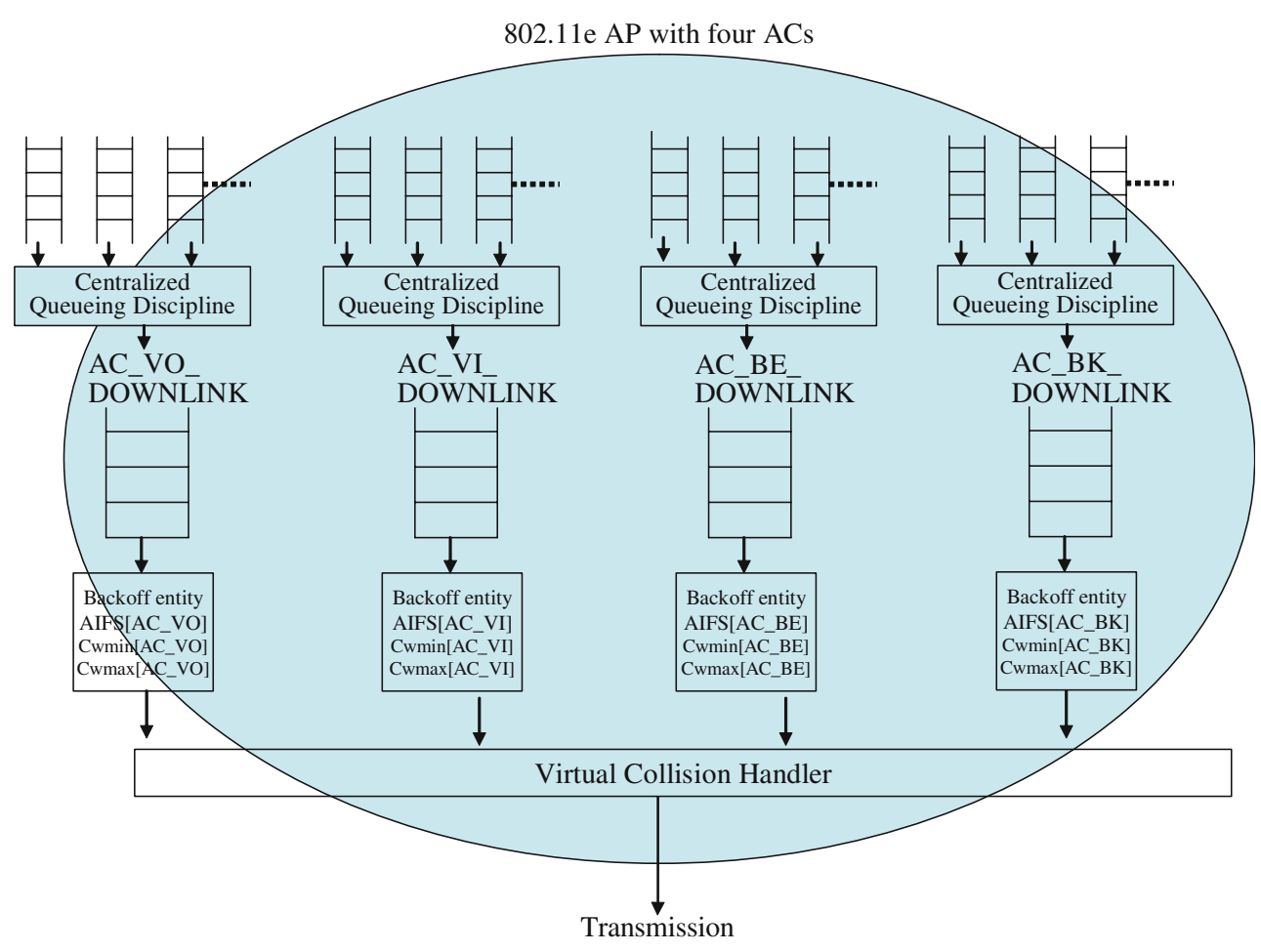

$h: i=\frac{n^{*} k}{j}$

Figure 3 gives an example of the bandwidth sharing hierarchy, where $C$ denotes the link capacity.

\section{Performance evaluation}

In this section, the performance of the 802.11e EDCA and DS-EDCA is evaluated with the ns-2 EDCA implementation created by Chesson and Singla [13]. The wireless link rate is set to $11 \mathrm{Mbps}$ and each data flow is associated with one $\mathrm{AC}$ and generates CBR UDP traffic at a rate of $8 \mathrm{Mbps}$ to the AP. The packets, including the IP header, of all ACs

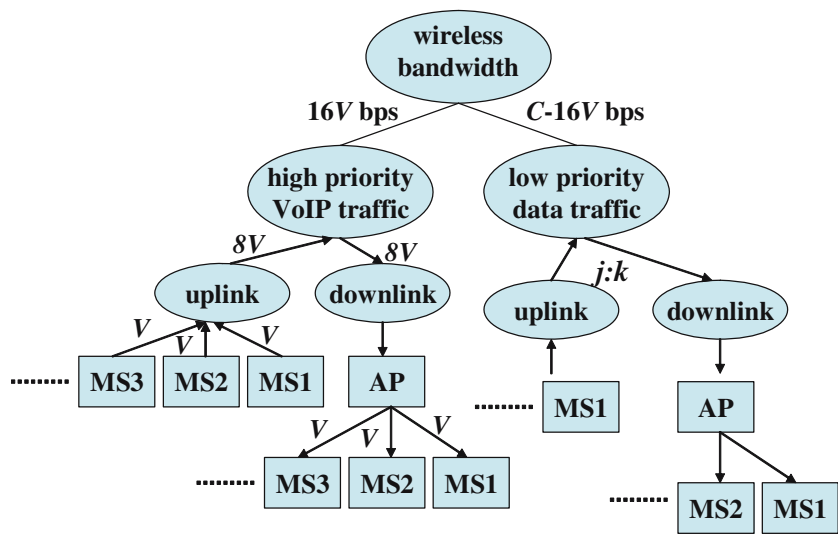

Figure 3 A hierarchical link sharing example are fixed at 1,520 B. The values of the EDCA parameter sets for the four ACs are listed in Table 1.

We first study the effect of strict priority implemented in our mechanisms for delay-sensitive traffic as compared to the original EDCA. In this simulation, only $\mathrm{AC}_{-} \mathrm{VO}$ and $\mathrm{AC}$ _VI flows are active. Figure 4 plots the average delay and delay variation of $\mathrm{AC} \mathrm{VO}$ as a function of the number of AC VI flows. It shows that the original EDCA is very sensitive to the number of active low priority flows, while in DS-EDCA, the average delays and delay variations are invariant to the traffic load of active low priority. This proves that the proposed mechanism is suitable to provide service for delay sensitive traffic such as VoIP over WLANs.

We then compare the proportional fairness provided by 802.11 EDCA and the proposed DS-EDCA under two different simulation scenarios. The weights of $\mathrm{AC} V \mathrm{~V}$, $\mathrm{AC}$ VI, AC_BE, and $\mathrm{AC} \mathrm{BK}$ are set to $0.4,0.3,0.2$, and 0.1 , respectively. The fairness is measured with respect to the fairness index FI defined in [14] as follows.

Table 1 Values of EDCA parameter sets used in the simulations

\begin{tabular}{lllll}
\hline & AC_VO & AC_VI & AC_BE & AC_BK \\
\hline AIFSN & 2 & 2 & 3 & 7 \\
CWmin & 3 & 7 & 15 & 15 \\
CWmax & 7 & 15 & 1,023 & 1,023 \\
\hline
\end{tabular}


Figure 4 The delay and delay variance of flows. a Average delay of AC_VO. b Delay variance of $\mathrm{AC}_{-}^{-} \mathrm{VO}$

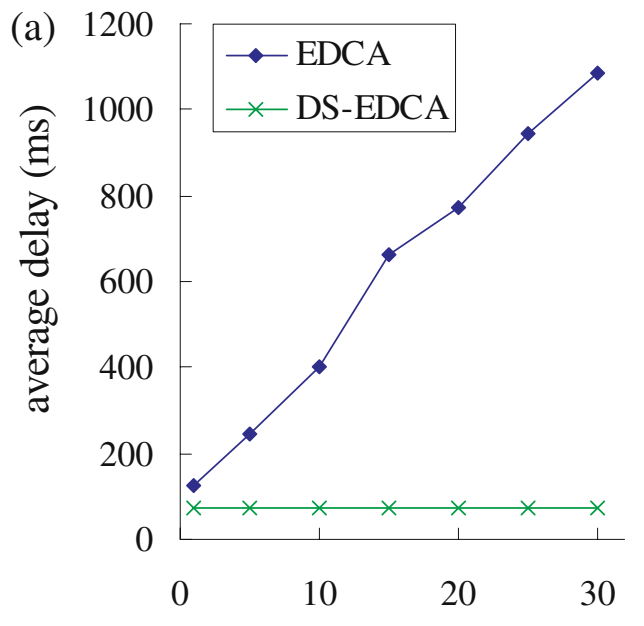

number of AC_VI flows

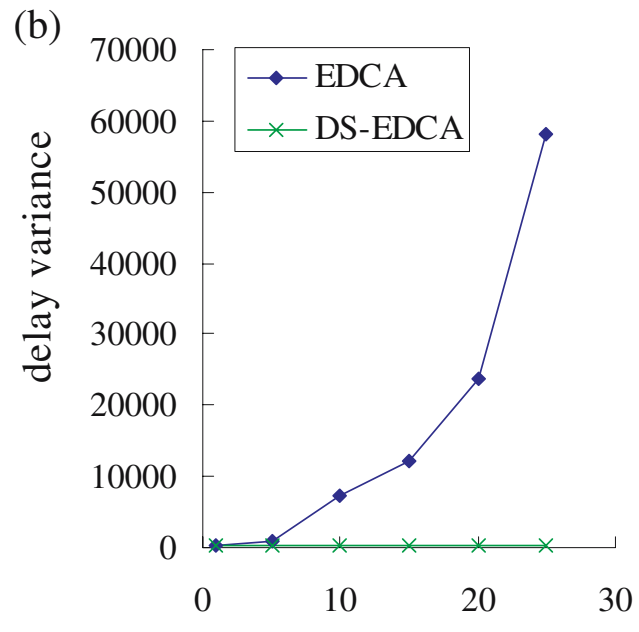

number of AC_VI flows
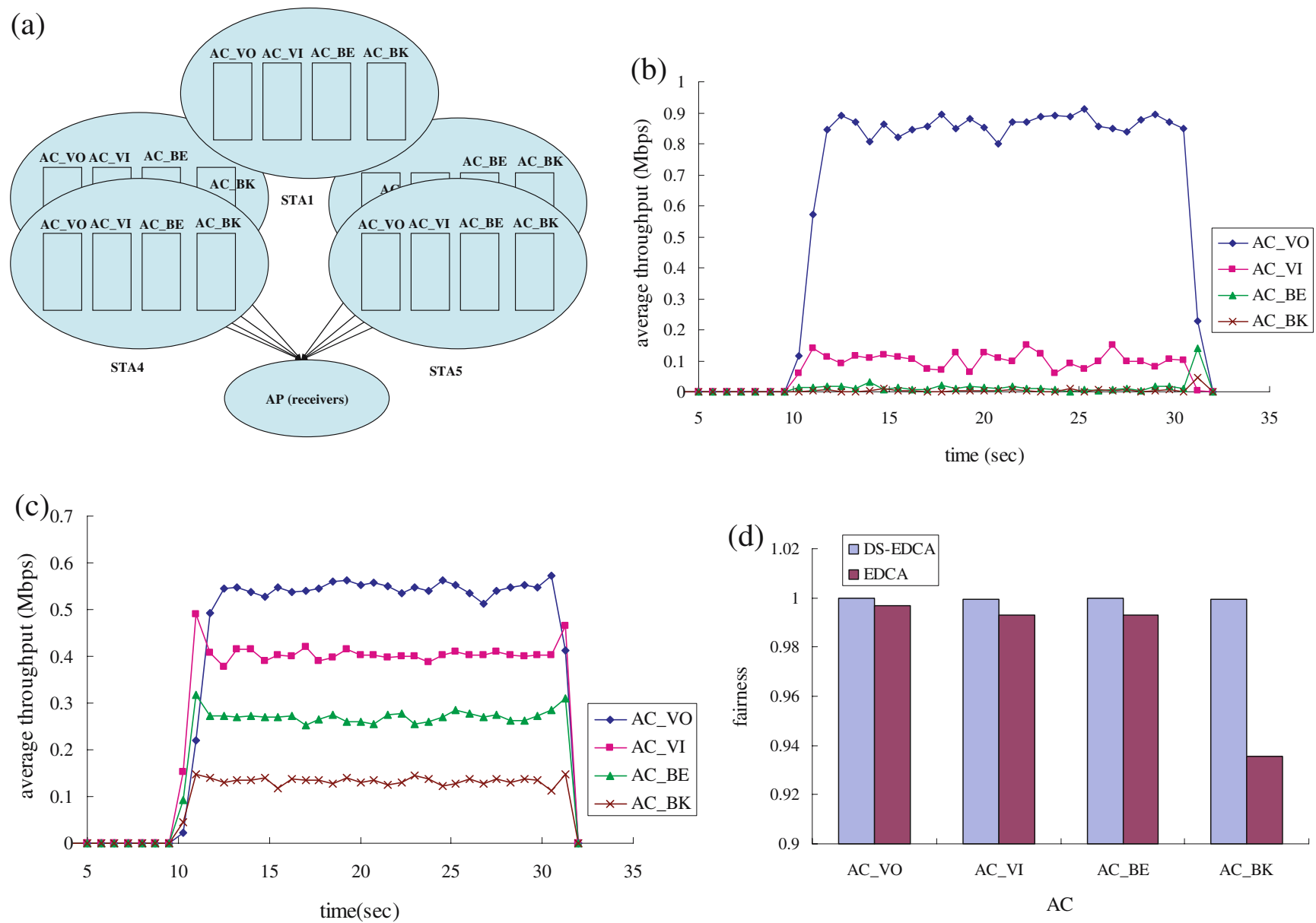

Figure 5 Scenario 1: 20 AC flows in five STAs and four flows in each STA. a Simulation topology. b EDCA (AC_VO: AC_VI: AC_BE: AC_BK $=176.95: 20.83: 3.4: 1 \quad \mathrm{FI}=0.36)$. c ${ }^{-} \mathrm{DS}-\mathrm{EDCA} \quad(\mathrm{AC}-\overline{\mathrm{VO}}$ :

AC_VI: AC_BE: AC_BK=4.05:3.04:2.03:1 FI=0.99). d The fairness indices among flows in the same $\mathrm{AC}$ 
$\mathrm{FI}=\frac{\left(\sum_{f} \frac{T_{f}}{\phi_{f}}\right)^{2}}{n \times \sum_{f}\left(\frac{T_{f}}{\phi_{f}}\right)^{2}}$,

where $T_{f}$ denotes the throughput of $\mathrm{AC}_{-} f$ and $n$ is the number of AC flows. The fairness index is a real value between 0 and 1 . The closer to 1 the fairness index, the fairer. In the first scenario, there are $20 \mathrm{AC}$ flows distributed in five STAs (i.e., with four flows in each STA) in Fig. 5a. We will show that DS-EDCA not only provides excellent fairness among flows of different ACs, but also achieves very good fairness for flows of the same $\mathrm{AC}$ in this scenario. In the second scenario, there are four AC flows distributed to two stations in Fig. 6a. We will demonstrate that even if not all ACs in one station are active, DS-EDCA can still provide weighted fair service to all active AC flows. We plot the average throughputs of different ACs in order to compare the fairness among ACs. We also compare the fairness among different flows for each AC (distributed in different stations). Figures 5 and 6 plot the aggregate flow throughput of each $\mathrm{AC}$ in the system for 802.11e EDCA and DS-EDCA (i.e., all subfigures (b) are for 802.11e EDCA, and (c) for DS-EDCA). In each sub-figure, we also identify the throughput ratios of AC_VO, AC_VI, AC_BE, and AC_BK flows with respect to $\mathrm{AC}$ BKK flows, and the fairness indices. Figure $6 \mathrm{~d}$ plots the fairness indices among flows of each AC for EDCA and DS-EDCA under the first scenario. We see that in all cases, with $802.11 \mathrm{e}$ EDCA, the high priority AC flows (i.e., AC_VO) consume most of the channel capacity such that it achieves poor fairness among flows of different ACs. DSEDCA, on the other hand, allows low priority AC flows to share channel capacity according to their assigned weights, thus providing proportional fairness nicely.

We now demonstrate the strength of the hierarchical link sharing architecture in 802.11e WLANs. In this simulation, we consider two pairs of G.711 VoIP calls (four uplink and four downlink VoIP flows), and four uplink and four downlink CBR UDP data flows, each operating at 4 Mbps. The objective of this simulation is to demonstrate that the link sharing model can guarantee QoS for VoIP sessions and ensure the aggregate throughputs of uplink and downlink data traffic in the ratio of 1:2. We configure the VoIP traffic and data traffic as $\mathrm{AC} \_\mathrm{VO}$ and $\mathrm{AC} \_\mathrm{BE}$, respectively, for the original $802.11 \mathrm{e}$ EDCA. In this simulation, only $\mathrm{AC} \_\mathrm{VO}$ and $\mathrm{AC} \_\mathrm{BE}$ flows are active. For the proposed hierarchical DS-EDCA, the downlink and uplink VoIP flows are configured as $\mathrm{AC} \_\mathrm{VO}$, and the weights of backoff entities for AP and all other mobile stations are set to $4: 1$. The downlink and uplink data flows
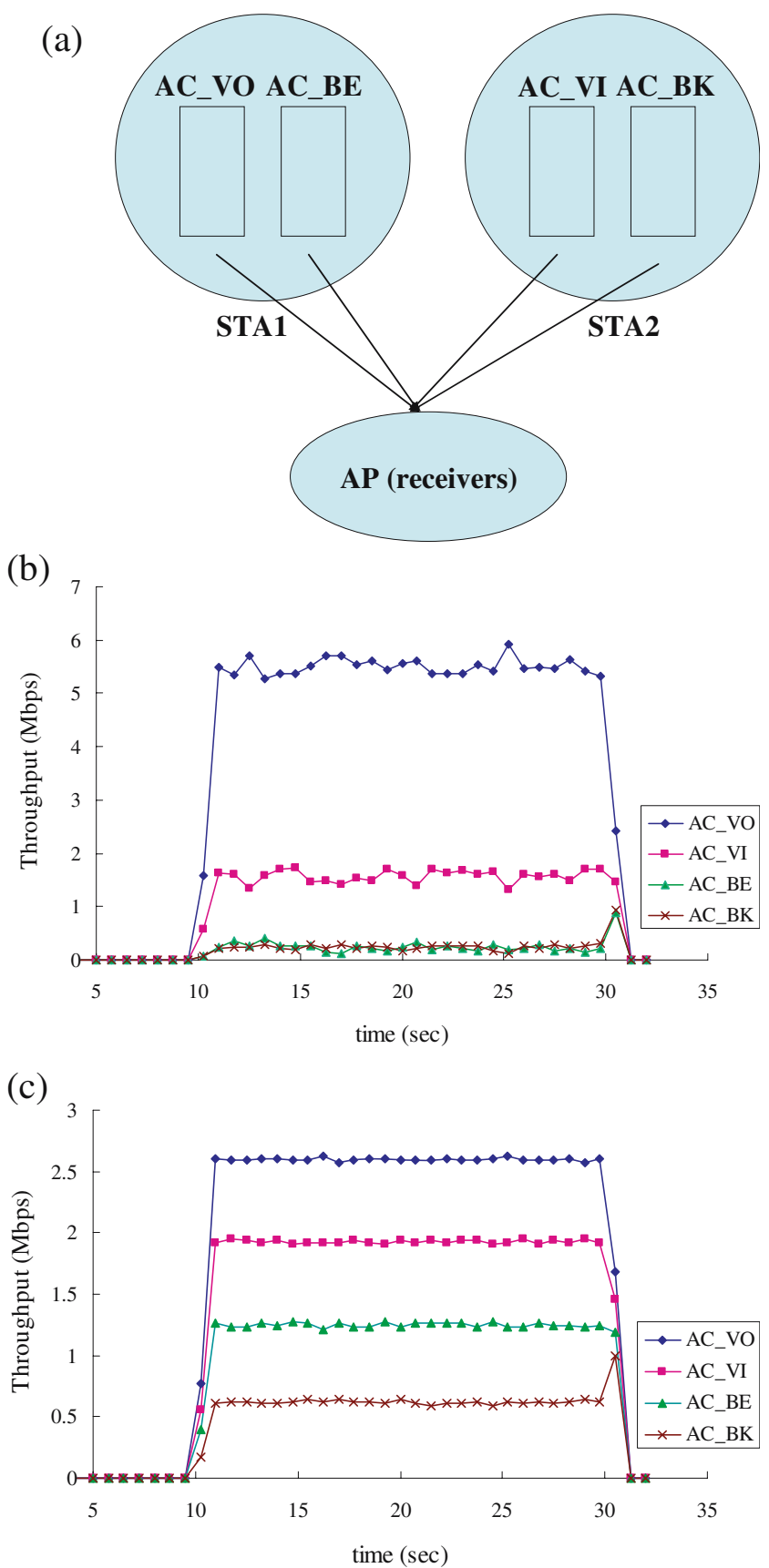

Figure 6 Scenario 2: four AC flows in two STAs; flows 1 and 3 in STA1, and flows 2 and 4 in STA2. a Simulation topology. b EDCA (AC VO: AC VI: AC BE: AC BK=22.97:6.58:1:1 FI=0.56). c DSEDCA (AC_V̄O: AC_VI: AC_BE: AC_BK=4.20:3.11:2.02:1 FI= 0.99 )

are all AC_BE, and the weights assigned to the backoff entities of $\mathrm{AP}$ and other mobile stations are set to $8: 1$ according to Eq. 10. AC_VO has strict priority over AC_BE.

Figure 7 shows the throughput performance of the original EDCA and DS-EDCA with different traffic types. Figure $7 \mathrm{a}$ and $\mathrm{b}$ plot the throughputs of VoIP flows for the original EDCA and DS-EDCA, respectively. DS-EDCA has a similar throughput variance of uplink VoIP flows as that 
(a)

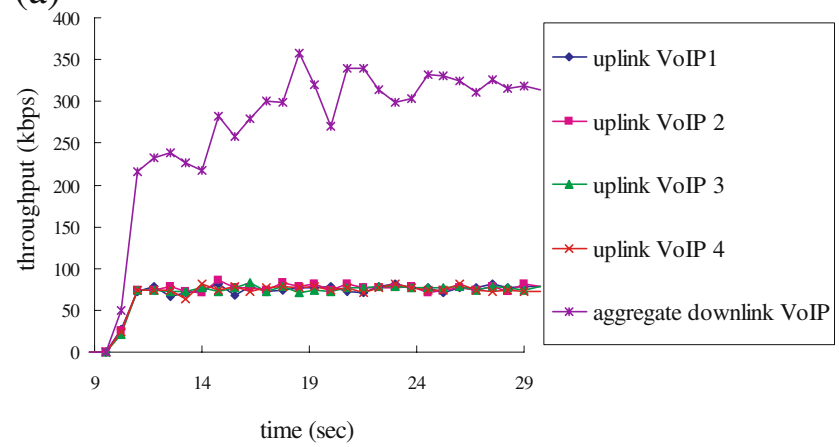

(c)

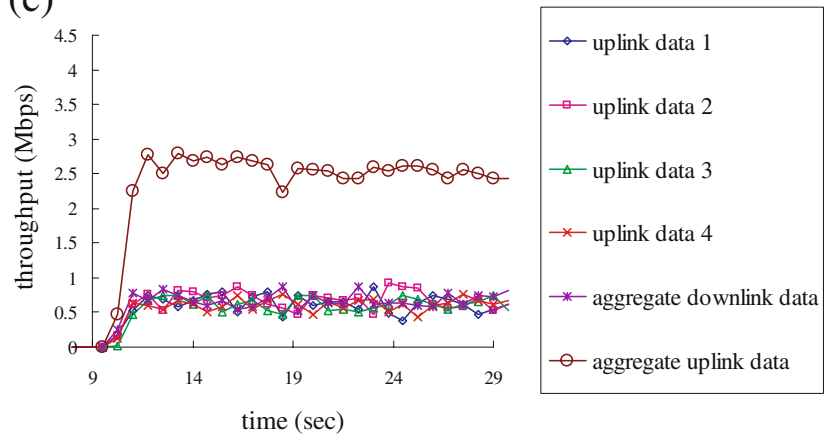

(b)

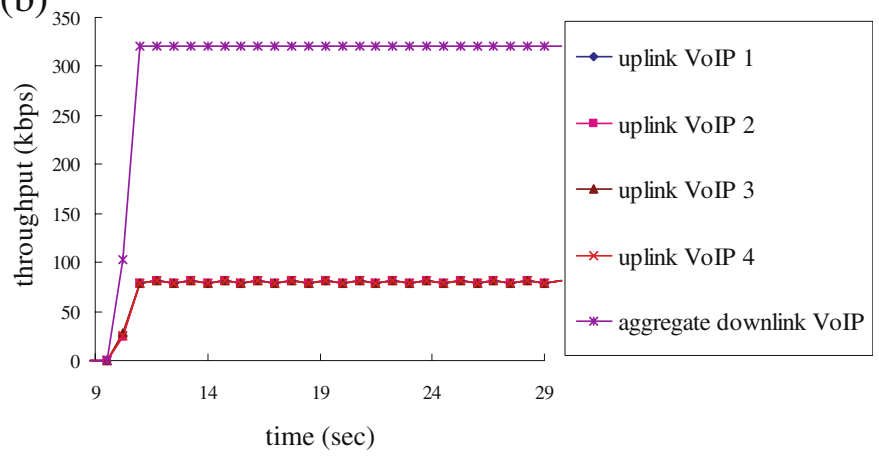

(d)

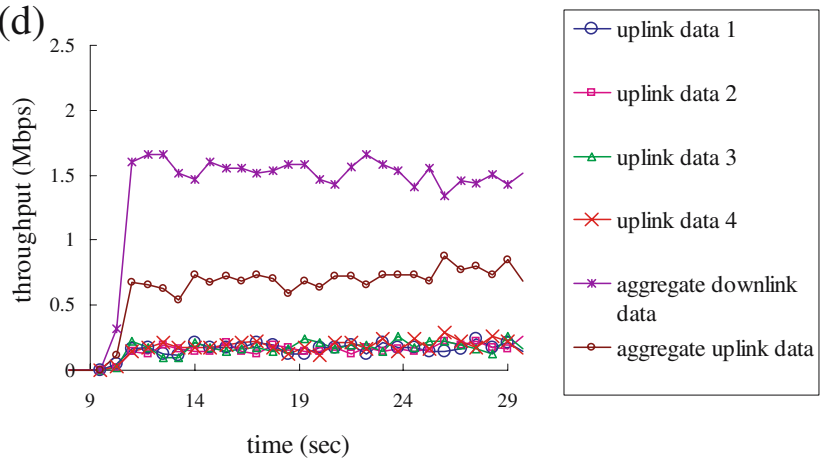

Figure 7 The throughputs of EDCA and DS-EDCA. a The throughputs of VoIP flows for EDCA. b The throughputs of VoIP flows for DS-EDCA. c The throughputs of data flows for EDCA. d The throughputs of data flows for DS-EDCA

in the original EDCA, but has a relatively stable and higher throughput for aggregate downlink VoIP traffic because the AP in the original EDCA does not have priority over mobile stations in channel contention. Thus, DS-EDCA can guarantee the demanded QoS for both uplink and downlink VoIP flows. Figure $7 \mathrm{c}$ and d plot the aggregate throughputs of data flows for EDCA and DS-EDCA, respectively. Figure $7 \mathrm{c}$ shows that the aggregate throughput of downlink data flows from the AP in EDCA is equal to that of each individual uplink data flow, which may lead to serious packet loss when traffic load is heavy. On the other hand, as shown in Fig. 7d, the aggregate throughput of downlink data flows is twice that of the aggregate uplink data flows in DS-EDCA, which fits the planned resource management policy (i.e., the ratio of uplink to downlink being 1:2). Moreover, each uplink data flow shares an equal amount of bandwidth, and thus the uplink resource is shared fairly. Therefore, the proposed service model can control the bandwidth sharing among downlink and uplink flows according to link sharing policy through the priority and weight assignments implemented in DS-EDCA.

\section{Conclusion}

In this paper, we have proposed a service mechanism called Differentiated Service-Enhanced Distributed Channel Access (DS-EDCA) for IEEE 802.11e WLANs. With DS-
EDCA, strict priority and weighted fair service among ACs are provided such that we can control the delay and delay variation of the QoS traffic and ensure proportional bandwidth sharing among the best-effort traffic. The EDCA parameter sets of lower priority traffic are carefully designed and the backoff intervals of ACs are determined according to DFS. Furthermore, a hierarchical link sharing model for IEEE 802.11e WLANs is proposed, which proves very useful in downlink and uplink traffic sharing. We have also conducted ns-2 simulations to compare the performance of IEEE 802.11e EDCA and DS-EDCA in terms of strict priority, proportional service, fairness, and aggregate throughput of each AC. The simulation results show that with DS-EDCA, the ACs can share the channel capacity strictly according to their priorities, assigned weights. Most importantly, DS-EDCA is easily implemented and is compatible with the IEEE 802.11 Standard.

\section{References}

1. International standard [for] information technology-Telecommunications and information exchange between systems - Local and metropolitan area networks - specific requirements-Part 11: Wireless LAN Medium Access Control (MAC) and Physical Layer (PHY) Specifications, Amendmenet 8: Medium Access Control (MAC) Quality of service enhancements, IEEE 802.11 WG, 2005 
2. Wi-Fi Alliance (2004) Wi-Fi Certified for WMMTM - Support for multimedia applications with quality of service in $\mathrm{Wi}-\mathrm{Fi}^{\circledR}$ networks

3. Parekh AK, Gallager RG (1993) A generalized processor sharing approach to flow control in integrated services networks: the single-node case. IEEE/ACM Trans Netw 1(3):344-357 (June)

4. Lee JF, Sun Y, Chen MC (2002) WF2Q-M: a worst-case fair weighted fair queueing with maximum rate control. IEEE GLOBECOM :1576-1580

5. Lu S, Nandagopal T, Bharghavan V (1998) A wireless fair service algorithm for packet cellular networks. ACM Mobicom :10-20

6. Vaidya NH, Bahl P, Gupta S (2000) Distributed fair scheduling in wireless 1AN. ACM Mobicom :167-178

7. Qiao Daji, Shin Kang G. (2002) Achieving efficient channel utilization and weighted fairness for data communications in IEEE 802.11 WALN under the DCF. ACM IWQoS :227-236

8. Pattara-Atikom Wasan, Banerjee Sujata, Krishnamurthy Prashant (2002) Starvation prevention and quality of service in wireless LANs. IEEE WPMC, Honolulu, HI, October 2002

9. Malli M, Ni Q, Turletti T, Barakat C (2004) Adaptive fair channel allocation for QoS enhancement in IEEE 802.11 Wireless LANs. IEEE ICC, Paris, France, July 2004

10. Chou CT, Shin KG, Shankar SN (2003) Inter-frame space (IFS) based service differentiation for IEEE 802.11 Wireless LANs. IEEE VTC

11. Golestani S (1994) A self-clocked fair queueing scheme for broadband applications. IEEE INFOCOM'94, Toronto, CA, June 1994

12. Shreedhar M, Varghese G (1996) Efficient fair queuing using deficit round-robin. IEEE/ACM Trans Netw 4(3):375-385

13. Available: http://ftp:Nftp-sop.inria.fr/rodeo/qni/ns-edcf.tar.gz

14. Jain R, Babic G, Nagendra B, Lam C (1996) Fairness, call establishment latency and other performance metrics. Tech. Rep. ATM_Forum/96-1173, ATM Forum Document, August

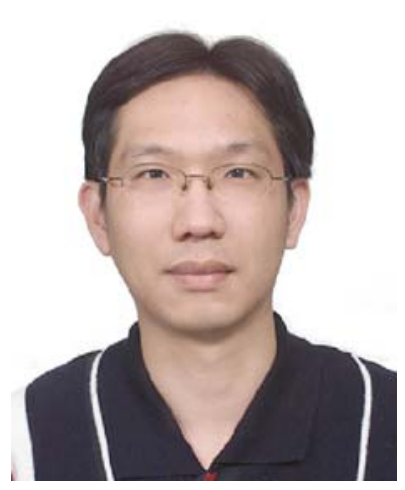

Jeng-Farn Lee received the B.S. and M.S. degrees in the Department of Information Management from National Taiwan University, Taiwan, in 1998 and 2000, respectively. He has been with Institute of Information Science, Academia Sinica, Taiwan as a research assistant since Jan. 2001. He is now a $\mathrm{Ph}$.D. candidate in the Department of Electrical Engineering, National Taiwan University. His current research interests include QoS networking, scheduling, and wireless access network.

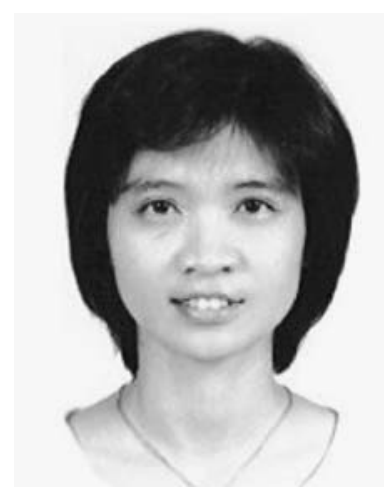

Wanjiun Liao received the B.S. and M.S. degrees from National Chiao Tung University, Taiwan, in 1990 and 1992, respectively, and the Ph.D. degree in Electrical Engineering from the University of Southern California, Los Angeles, CA, USA, in 1997. She joined the Department of Electrical Engineering, National Taiwan University (NTU), Taipei, Taiwan, as an Assistant Professor in 1997. Since August 2005, she has been a full professor. Her research interests include wireless networks, multimedia networks, and broadband access networks.

Dr. Liao is actively involved in the international research community, and has served on the program committees of many international conferences. She was an editor of Computer Networks, and is currently an editor of IEEE Transactions on Wireless Communications and IEEE Transactions on Multimedia. Dr. Liao has received many research awards. Two papers she co-authored with her students received the Best Student Paper Award at the First IEEE International Conferences on Multimedia and Expo (ICME) in 2000, and the Best Paper Award at the First International Conference on Communication, Circuits and Systems (ICCCAS) in 2002. Dr. Liao was elected as one of Ten Outstanding Young Women in Taiwan in 2000 and was listed in the Marquis Who's Who in Science and Engineering.

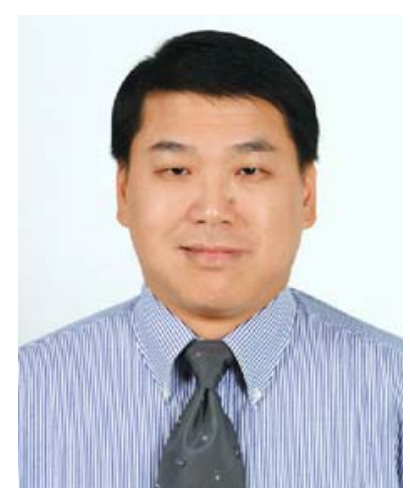

Meng Chang Chen received the B.S. and M.S. degrees in Computer Science from National Chiao-Tung University, Taiwan, in 1979 and 1981, respectively, and the Ph.D. degree in Computer Science from the University of California, Los Angeles, in 1989. He joined AT\&T Bell Labs in 1989 as Member of Technical Staff and led several projects in the area of data quality of distributed databases for mission critical systems. He was an Associate Professor at Department of Information Management, National Sun Yat-Sen University, Taiwan, from 1992 to 1993. Since then he has been with Institute of Information Science, Academia Sinica, Taiwan. He has held Associate Research Fellowship since July 1996, and assumed the responsibility of Deputy Director from August 1999 to July 2002. His current research interests include QoS networking, wireless network, multimedia systems and transmissions, operating systems, and data and knowledge engineering. 